\title{
Angular segregation of fibres in pipe flow: floc formation and utilization for length-based fibre separation
}

\author{
Jakob D. Redlinger-Pohn
}

Received: 27 February 2020 / Accepted: 12 June 2020 / Published online: 4 July 2020

(C) The Author(s) 2020

\begin{abstract}
Cellulose fibres are prone to flocculate and form aggregates that are deformable by the hydrodynamic stress. In this work I document for coiled pipe flow, known to have secondary motion in the pipe cross-sectional plane, an accumulation of fibre flocs and fibre aggregates at the outer bend. That is the segregation into a section in the pipe cross-section and hence presents a case of angular segregation. The segregation was studied for non-coherent crowded fibre flocs. For that, segregation benefited from fibre concentration and suffered from increased hydrodynamic stress expressed by increasing Reynolds number. Based on the observed segregation of fibres a flow splitter was designed that separated the flow at $1 / 3$ of the tube diameter measured from the inner bend. The outer bend suspension length-weighted fibre length was found to increase. For the best case in this work,
\end{abstract}

Electronic supplementary material The online version of this article (https://doi.org/10.1007/s10570-020-03290-8) contains supplementary material, which is available to authorized users.

J. D. Redlinger-Pohn ( $\square)$

Department of Engineering Mechanics, KTH Royal Institute of Technology, Teknikringen $8 \mathrm{~F}$,

11428 Stockholm, Sweden

e-mail: edu.redlinger@gmail.com

J. D. Redlinger-Pohn

Institute of Process and Particle Engineering, Graz University of Technology, Inffeldgasse 13, 8010 Graz, Austria the difference between outer and inner bend relative to the feed fibre length was $22 \%$. As for radial and axial segregation, which are known, also angular segregation is fibre-length sensitive. As such it can be exploited for length fractionation of networking and aggregating elongated particles, for example fibres.

Keywords Fibre segregation - Coiled flow · Flocculation · Fractionation · Length-based fibre separation $\cdot$ Size separation

\section{Introduction}

Highly elongated particles, for example, cellulose fibres, flocculate and form a porous network from elastic interlocking (Kerekes et al. 1985; Soszynski 1987; Soszynski and Kerekes 1988a, b): cohesion from fibre-fibre friction exceeds dispersion, for example from hydrodynamic stress. Whilst the network of flocculated fibres can withstand dispersion at moderate stress, it can undergo deformation (Meyer 1964). The fibre network in suspension flow through straight pipes was found consolidating towards the centre what yields a near-wall annulus free of fibres. Steenberg and Wahren (Steenberg and Wahren 1960) found that this annulus contains small particles, i.e. fibre-fines, that are not coherently incorporated in the fibre network. This describes the case of radial fibre 


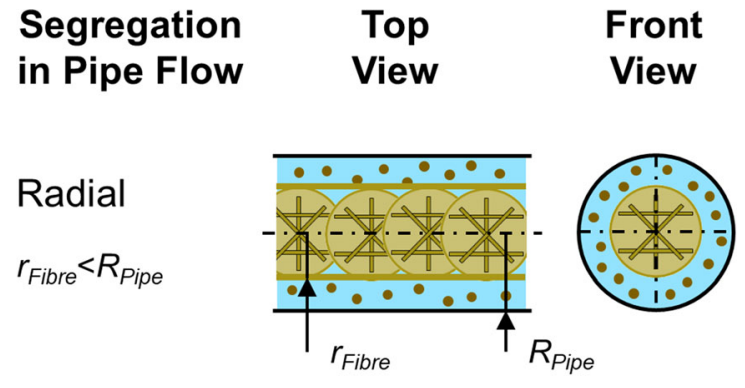

Axial

Compartment in flow direction z

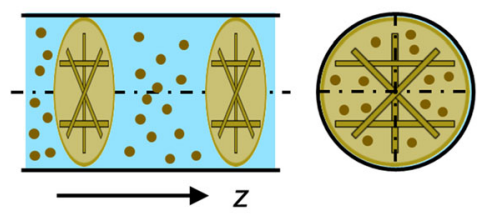

Angular

$$
\varphi_{\text {Fibre }}<2 \pi
$$
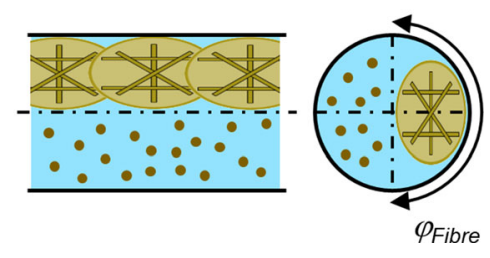

Fig. 1 Illustration of fibre segregation in pipe flow as seen from the top and front view. First row: radial fibre segregation in annular plug flow. Fibres are confined to a consolidated network smaller than the tube radius, i.e. $r_{\text {Fibre }}<R_{\text {Pipe }}$. Fibre-fines are present in the firbes free area, i.e. $>r_{\text {Fibre }}$. Second row: axial fibre segregation. Fibres and fibre-fines appear in alternating compartments in flow direction $z$. Third row: angular fibre segregation. Fibres segregate to a segment in the tube crosssection denoted by an angle $\varphi_{\text {Fibre }}<2 \pi$

segregation by the fibre length. An illustration is given in Fig. 1 first row, with long fibres consolidated to a radius $r_{\text {Fibre }}$ smaller than the pipe radius $R_{\text {Pipe }}$. Fibrefines (i.e. particles smaller than $200 \mu \mathrm{m}$ (ISO 16,065-2)) are suspended in the pipe cross-section. Redlinger-Pohn et al. (2017a,b) successfully utilized the flow situation for fibre-length fractionation. In a process termed hydrodynamic fractionation, they removed the near-wall suspension essentially free of long fibres by side-channels and hence separated fibre-fines exclusively.

Axial segregation, illustrated in Fig. 1 second row, is another example of length-based fibre segregation from fibre crowding and network deformation. In principle, it describes the flow of long and short fibres (or fibre-fines) in consecutive compartments, documented by Johansson and Kubát (1956) for fibre suspension slug flow. Within the liquid slug, a secondary motion, that is parallel to the flow direction, arises. The secondary motion is in flow direction at the pipe centre and counter flow direction at the pipe wall. Suspended material is transported to the slug front, i.e. after the bubble, where fibres flocculate and form a plug. Fibre-fines are mixed in the liquid slug and hence tail the long fibres in the transport direction. The principle was utilized commercially for industrial length-based fibre fractionation, for example in the Johnson fractionator (Olgard 1970; Olgard and Axenfalk 1972).

Following the nomenclature of cylindrical coordinates, the question arises on the existence of angular segregation, i.e. segregation and preferential accumulation of fibres in a circular segment of the tube crosssection, i.e. $\varphi_{\text {Fibre }}<2 \pi$, illustrated in Fig. 1 third row. In fact, angular segregation of long fibres was hinted on by Redlinger-Pohn et al. (2016a; b) being the leading mechanism in tube flow fraction. Tube flow fractionation is a commercial preparation technique of cellulose pulp for analytics from Metso (Metso Automation Oy, Finland, (Niinimäki et al. 2007)). The fractionator was introduced by Silvy and Pascal as an auxiliary unit for sample preparation as a "tube of a few hundred meters long which may be rolled up to avoid bulkiness" (Silvy and Pascal 1990). A fibre pulp sample injected into a flow through the coiled tube separated the fibres by their length: long fibres exit the tube first, followed by short fibres and fibre-fines (Laitinen et al. 2006, 2011). That separation result is comparable to axial segregation (compare to Fig. 1 second row Johansson and Kubát (1956)) which led to confusion on the separation mechanism in the literature (Krogerus et al. 2003). The argument based on the outflow results ignores a fundamental difference between slug flow and coiled pipe flow: the secondary motion is parallel to the flow direction in slug flow (Talimi et al. 2012) and normal to the flow direction in coiled-pipe flow (Dean 1927; Vashisth et al. 2008). The cross-sectional mixing flow or secondary motion arises from a pressure imbalance between the outer and inner bend of the coiled pipe resulting from centrifugal forces. In addition, the velocity maximum is deflected from the pipe centre to the coiled-pipe outer bend. The secondary motion is known to (i) resuspend non-flocculating particles (Koutsky and Adler 1964; Palazoglu and Sandeep 2004) (ii) enhance mixing (Naphon and Wongwises 2006) and (iii) promote colloidal flocculation of polymers (Carissimi et al. 2018). Redlinger-Pohn et al. (2016a; b) 
compared numerical results of individual fibres transported in the coiled pipe flow to experiments with cellulose fibre samples and argued from differences, that long fibres in coiled pipe flow flocculate. The observed length-separation at the outflow was addressed to differences in the networking capabilities of long and short fibres. The separation quality of $0.3 \mathrm{~mm}$ and $4 \mathrm{~mm}$ fibres was tested for concentrations yielding non-coherent and coherent flocs. The separation was unsatisfactory for a fibre sample yielding coherent flocs. Further, they documented a decreasing residence time of fibre length fractions with a increasing fibre concentration $C$ (cohesion) and an decreasing Reynolds number $R e$ (dispersion). That agrees with a recommendation in Krogerus et al. (2003) to operate the tube flow fractionator with concentrations below the threshold for coherent flocs. An indication of angular segregation was found by Jagiello et al. (2016) who split the coiled tube outflow in an inner and outer bend flow. The outer bend flow mean fibre length $l_{l}$ was larger compared to the inner bend flow with a negative dependence of the length difference on the Reynolds number Re. But doubt remained on the segregation mechanism for two reasons: (i) an opaque tube prevented visual observation, and (ii) the earlier mentioned confusion in literature.

With hindsight on the increased demand for separated fibre and fibre-fines streams for the production of paper and board with dedicatedly set and tuned properties (Odabas et al. 2016; Mayr et al. 2017; Fischer et al. 2017; Bossu et al. 2019), angular segregation and its potential for length-based fibre separation should be re-addressed. Commonly applied pressure screening relies on the individual treatment of each particle, i.e. fibre or fibre-fines, and invests energy to disperse the networked fibres (an overview on pressure screening is for example given by Jokinen (2007)). Processes that can utilize fibre flocculation and fibre networking avoid the need for this extra energy (Olgard and Axenfalk 1972; Redlinger-Pohn et al. 2017a, b; Schmid et al. 2019).

In this paper I will provide visual evidence of angular segregation from coiled suspension flow. This insight and understanding are then transferred into a prototypical separation process. The results are promising and present a simple and straight-forward way of separating fibre pulp by the fibre length.
The paper is organized as follows. In Section 2 I describe the setting for the visual observation and fibre pulp separation. In Section 3 I present the results from both studies. In Section 4 I elucidate the fibre segregation in coiled suspension flow. In Section 5 I conclude the study. The Appendix adds further comments on the observed fibre segregation and contains scaling analysis mentioned in the discussion. The Supplement Material shows the high-speed recordings of flocculating fibres in coiled tube flow.

\section{Materials and methods}

The choice of fibre was taken with hindsight on the optical investigation. Dyed fibres have a larger contrast and are less transparent. For simplicity and from availability, mono-coloured napkin tissue paper (Aro Napkins, Metro whole-seller shop brand) was reslushed after soaking in water for a minimum of $24 \mathrm{~h}$. Availability (in the institute's social room) led to the use of two types of coloured napkins: blue and red. Blue was used for video observation and red for the length-based fibre separation. The properties of the fibre pulp from re-slushed napkins were determined with a L\&W Fibre Tester Plus (ABB, Sweden). The mean properties are listed in Table $1 . l_{l}$ is the lengthweighted and $l_{3}$ is the volume-weighted mean fibrelength.

The fibre interaction is characterized by the crowding number $N_{C W}$ (Kerekes and Schell 1992):

$N_{C W}=5\left[\frac{k g}{m^{3}}\right] \frac{C l_{1}^{2}}{c s}$

$c s$ is the average line density of the fibre (coarseness in pulp and paper nomenclature) and $C$ the mass-based fibre concentration in [\%]. Kerekes and Schell (1992) have shown, that coherent networks form for $N_{C W}$ $\geq 60$ (for a length to diameter aspect ratio $A R>20$ ).

Table 1 Length-weighted $\left(l_{1}\right)$ and volume-weighted $\left(l_{3}\right)$ mean fibre length and average fibre coarseness $c s$ as measured with the L\&W Fibre Tester Plus (ABB, Sweden)

\begin{tabular}{llll}
\hline & $l_{1}[\mathrm{~mm}]$ & $l_{3}[\mathrm{~mm}]$ & $c s[\mathrm{mg} / \mathrm{m}]$ \\
\hline Blue & 1.901 & 2.061 & 0.177 \\
Red & 1.553 & 1.783 & 0.118 \\
\hline
\end{tabular}


Between $16<N_{C W}<60$, fibres aggregate and form flocs that are more easily dispersible (Kerekes 2006). For $N_{C W}<16$, fibres interact without flocculation. The concentration in the experiments corresponded to $N_{C W}$ ranging from 4 to 20 . That is within the optimum of $N_{C W}<60$ (Krogerus et al. 2003) and non-coherent flocculation can be assumed.

The experimental setup, sketched in Fig. 2, followed work of Redlinger-Pohn et al. (2016a) and Jagiello et al. (2016) and consisted of a transparent tube, $D_{\text {Tube }}=16 \mathrm{~mm}$, coiled at a diameter of $D_{\text {Coil- }}$ $=400 \mathrm{~mm}$. The curvature, $\kappa=D_{\text {Tube }} / D_{\text {Coil }}$, calculates to 0.04 . The coordinate $h$ runs along the coil radius $R_{\text {Coil }}$ from the tube inner bend to the tube outer bend.

Greyscale images of the suspension flow were recorded after 1.5 coils from the inlet $(0.25$ coils from the outlet) at a frequency of $500 \mathrm{~Hz}$. Low transmission and higher grey value correspond to higher fibre concentration. The light absorption is expressed as time-averaged relative light absorption $R L A$ and calculated as the grey image compliment compared to the maximum of 255 . The $R L A$ was calculated as

\section{Top View Coiled Tube}

$$
R_{\text {Coil }}=200 \mathrm{~mm} . \kappa=0.04 \text {. }
$$

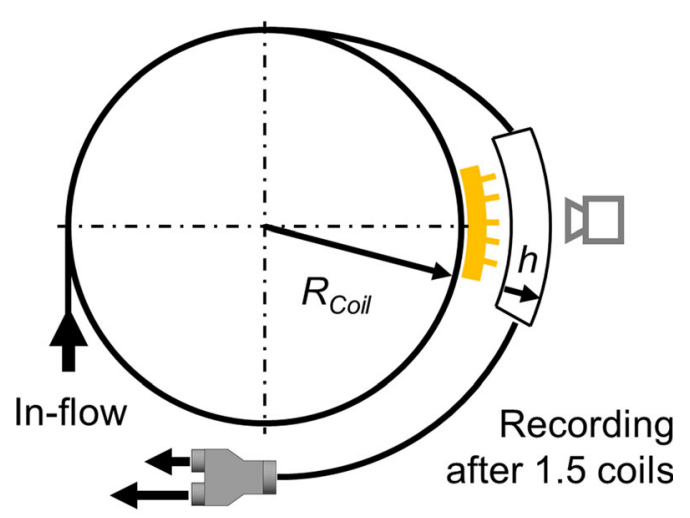

Flow splitter: Inner and outer bend flow

Fig. 2 Experimental set-up: a transparent tube $D_{\text {Tube }}=16 \mathrm{~mm}$ coiled at a diameter $D_{\text {Coil }}=400 \mathrm{~mm}\left(R_{\text {Coil }}=200 \mathrm{~mm}\right)$. The curvature $\kappa=0.04$. The light transmission through the fibre suspension is recorded 1.5 coils from the inlet. The sample is split into an inner bend and outer bend flow after 1.75 coil. $h$ is a coordinate in the direction of the coil radius from the tube inner to the tube outer bend mean over 200 recorded images along $h$. The recorded high-speed videos are presented in the Supplement Material.

The suspension flow was split into an inner and outer bend flow after 1.75 coils from the inlet. The prototypical flow splitter was 3D printed in-house. The design was guided by results from the optical investigation. Details are hence presented in the results section.

A typical metrics to report the condition of a coiled flow is the Dean number Da (see for example (Dean 1927; Itō 1959; Naphon and Wongwises 2006; Vashisth et al. 2008)):

$D a=\operatorname{Re} \sqrt{\kappa}$, with

$\operatorname{Re}=\frac{U_{\text {Bulk }} D_{\text {Tube }}}{v}$

which characterizes the effect of inertial, viscous, and centrifugal forces on the flow. $R e$ is the Reynolds number, $v$ the kinematic viscosity, and $U_{B u l k}$ the average stream-wise velocity. Canton et al. (2017) however showed in a recent and dedicated work that different flow conditions exist for the same $D a$ but different combinations of $R e$ and $\kappa$. The experimental settings in this work are hence reported with their Reynolds number $R e$ and curvature $\kappa$.

\section{Results}

Observation of fibre crowding in coiled suspension flow

Figure 3 provides a visual of the fibre suspension motion in coiled suspension flow observed from a top view. The case fibre concentration $C$, corresponding crowding number $N_{C W}$, and Reynolds number $R e$ (based on the mean flow rate, $D_{\text {Tube, }}$ and the viscosity of water as a reference, $v=10^{-6} \mathrm{~m}^{2} / \mathrm{s}$ ) are stated for each case. $N_{C W}$ is calculated according to Eq. 1 with values for Blue (Table 1).

Recordings in Fig. 3 capture fibre segregation to the outer tube bend and the formation of flocs already at a low crowding number of $N_{C W}=8$. The fibre crowding benefits from the fibre concentration $C$ and suffers from increased hydrodynamic stress, i.e. higher Reynolds number $R e$. The time-averaged $R L A$ profile along $h$ (from the inner to outer tube bend) is shown in 


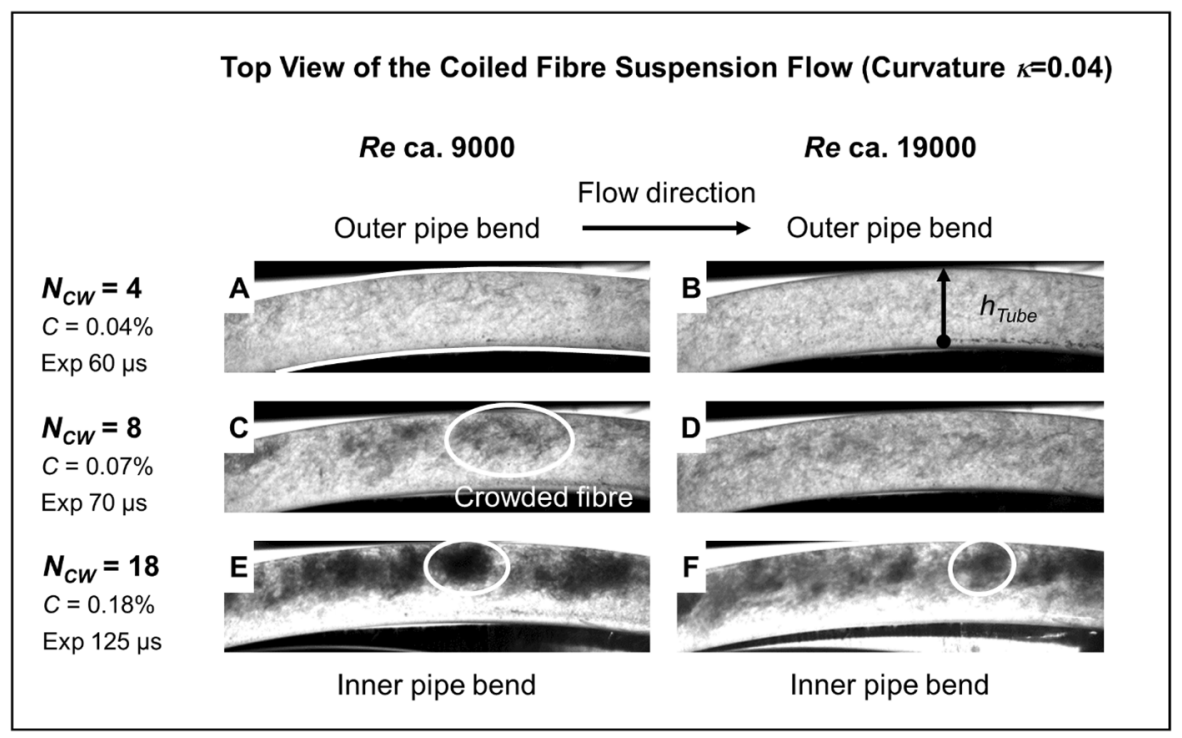

Fig. 3 First frame of each case recorded video (full video in the Supplement Material). The images state the case label letter (af). The flow direction is from the left to the right of the image. The light absorbance, i.e. higher grey value, corresponds to the local fibre concentration $C$. The exposure time, fibre concentration $C$, crowding number $N_{C W}$ and Reynolds number $R e$ is

Fig. 4, at a position, indicated in Fig. 3B. The error bar is 5 times the standard deviation of the $R L A$ in time and hence an indicator of the local fibre crowding. $R L A$ standard deviation is high for large temporal (or in stream-wise direction) differences in the local fibre concentration.

At $N_{C W}=4$ (Fig. 4a) $R L A$ was nearly constant with a slim increase towards the outer bend for $R e=9000$. The fibres were well distributed in the tube. At $N_{C W}=8$ (Fig. 4b) $R L A$ increases between $h=0.2$ to $h=0.7$ from the inner bend to the outer bend at $R e=9000$. Fibres were segregated to the outer bend. The difference between outer and inner bend was significantly lower at $R e=19,000$. That documents a dispersion of fibre aggregates at increased hydrodynamic stress in the coiled tube flow. At $N_{C W}=18$ (Fig. 4c) RLA was significantly higher at the outer bend for both $R e$ cases. The inner bend area was however over-exposed, and a small concentration of fibres may have been concealed by the bright background. Noteworthy is a subtle difference from increased $R e$ at $N_{C W}=18$ (Fig. 4c): $R L A$ at the outer bend was slightly decreased. Also in Fig. $3 \mathrm{f}$ bright spots that indicate fibre depletion can be identified what differs from the situation at lower Re (Fig. 3e). stated for each case. Local fibre crowds or aggregates are indicated by a white frame. $\mathbf{b}$ evaluation position for the timeaveraged light absorption shown in Fig. 4. e and $\mathbf{f}$ the inner pipe bend area is bright from over-exposure. The exposure time was adjusted to the higher concentration at the outer bend

This difference will be addressed in the discussion. The RLA standard deviation is low for $N_{C W}=4$ (Fig. 4a). The fibres were well-dispersed also in stream-wise direction and no flocculation of significance was observed (Fig. 3a, b). The RLA standard deviation is also low for $N_{C W}=18$ (Fig. 4c). Fibres were observed flocculated and segregated to the outer bend. The local fibre concentration with time (or in stream-wise direction) was low. An exception is the outer bend area at $R e=19,000: R L A$ is not only decreased but also varying in time. The $R L A$ standard deviation is comparatively high for $N_{C W}=8$ (Fig. $4 \mathrm{~b}$ ). At $R e=9000$, fibres were observed aggregating into distinguishable flocs (Fig. 3c) with an increasing $R L A$ towards the outer bend (Fig. $4 \mathrm{~b}$, grey line). The $R L A$ standard deviation is large at the inner bend which suggests dynamic local fibre flocculation and dispersion. Figure $3 \mathrm{c}$ documents some of the fibre aggregates segregated to the outer bend appearing stretched towards the inner bend. That will be addressed in the discussion.

The fibre suspension flow through the coiled tube from observation (videos are included in the Supplementary Material) is described in detail in the Appendix, Table 3. 
Relative Light Absorption RLA (Grey Intensity)

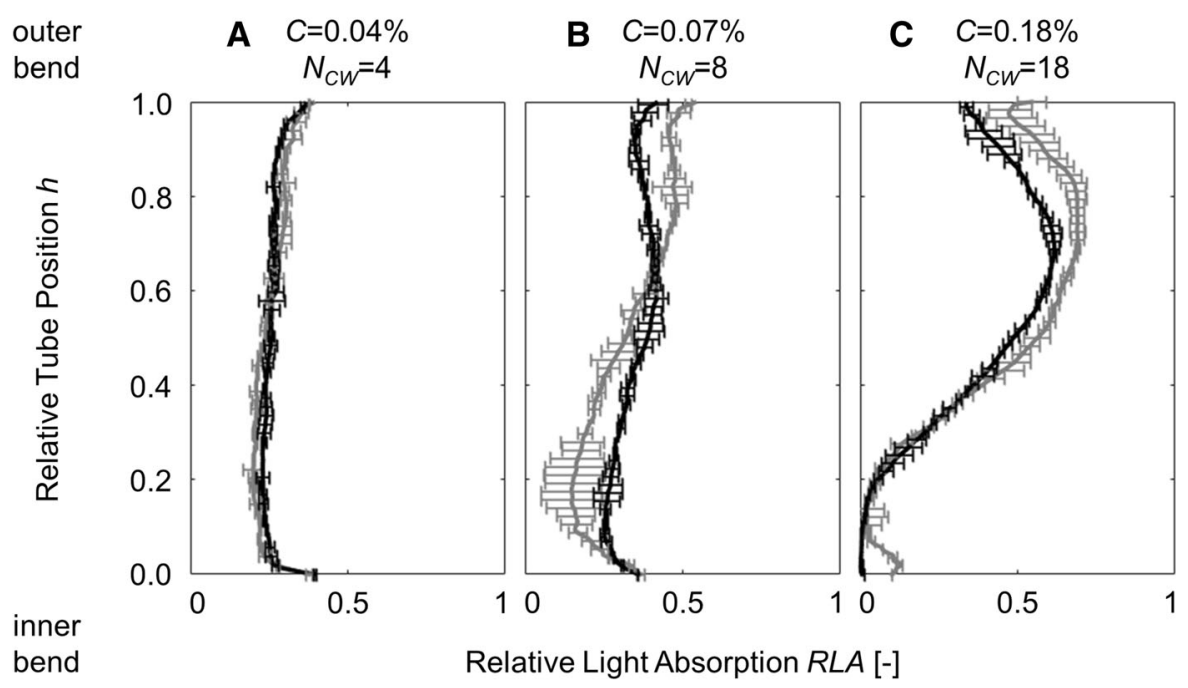

Fig. 4 Relative light absorbance along $h$ for cases presented in Fig. 3. $R e=9000$ in grey and $R e=19,000$ in black. a $C=0.04 \%, N_{C W}=4$. b $C=0.07 \%, N_{C W}=8$. c $C=0.18 \%$,

Length-based separation of fibre pulp

Observations of the suspension flow showed segregation of flocculated fibres to the outer bend, i.e. $h>0.3$. The flow splitter was designed accordingly and separated the inner from the outer bend flow at $h=D_{\text {Tube }} / 3$. The prototypical flow splitter as realized by $3 \mathrm{D}$ printing is presented in Fig. 5. Two areas are highlighted: the splitter blade in red and an area of observed flow recirculation in orange.

A splitter blade, i.e. a sharp edge in flow direction, is known to accumulate fibres by stapling. Kerekes et al. (1985) for example used it as floc generator, and Eß1 (2017) showed that even openings larger than the fibre length can clog from stapling at low flow speed. The area of the inner bend section is smaller than the outer bend section, and the velocity is higher at the outer bend (high velocity counters stapling. See for example (Eß1 2017)). Speculative, stapled fibres could impact the ratio of flow split and change the separation quality by being re-entrained at the outer bend or inner bend flow. The impact of the splitter blade on the fibre separation quality was not attained in this proof-ofconcept. For the inner bend outflow, the expansion led to a region of lower pressure and a recirculation which impacted the upstream flow. The test settings and average results for the separation proof-of-concept
$N_{C W}=18$. The error bar is 5 times the standard deviation in $R L A$ and presents a fluctuation of the local fibre concentration with time

\section{Flow Splitter}

Fines rich

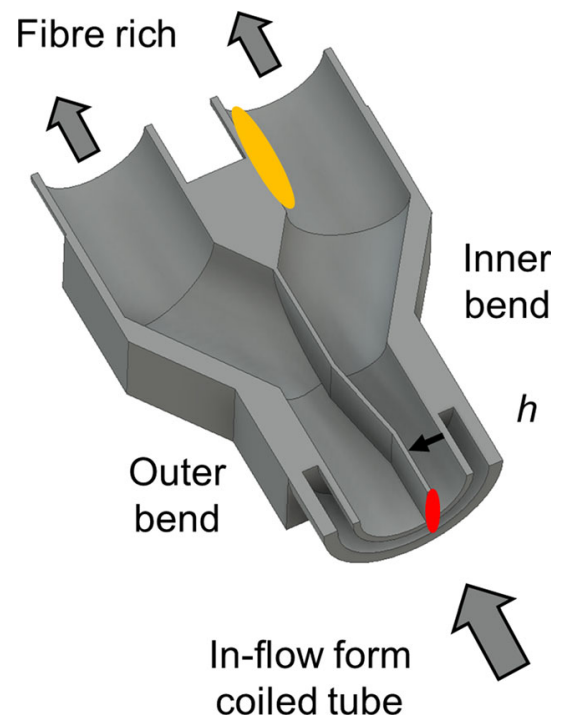

Fig. 5 CAD model cut for $3 \mathrm{D}$ printing of the flow splitter. The inner bend suspension flow was split from the outer bend suspension flow at $h=D_{\text {Tube }} / 3$. The yellow area marks a zone of lower pressure and observed fluid recirculation. The red area marks the flow splitter which is prone to fibre stapling 
Table 2 Separation results from splitting the coiled pipe flow. $\Delta l_{1, \text { rel }}$ is the difference of the outer bend to inner bend $l_{l}$ relative to the feed $l_{1}$, Feed

\begin{tabular}{llll}
\hline Separation & 1 & 2 & 3 \\
\hline Figure 3 & $\mathrm{D}$ & $\mathrm{F}$ & - \\
$\operatorname{Re}[-]$ & 19,436 & 19,206 & 25,356 \\
$N_{C W}[-]$ & 11 & 21 & 20 \\
$l_{l, \text { Feed }}[\mathrm{mm}]$ & 1.575 & 1.514 & 1.473 \\
$l_{l, \text { inner }}[\mathrm{mm}]$ & 1.518 & 1.247 & 1.343 \\
$l_{l, \text { outer }}[\mathrm{mm}]$ & 1.587 & 1.582 & 1.605 \\
$\Delta l_{1}[\mathrm{~mm}]$ & 0.069 & 0.335 & 0.262 \\
$\Delta l_{l, \text { rel }}[\%]$ & 4 & 22 & 18 \\
\hline
\end{tabular}

study are listed in Table 2. The first two settings correspond to case D (Fig. 3d) and case F (Fig. 3f).

The results of the separation study (Table 2) agree well with the visually observed fibre segregation (Fig. 3). Separation $1, N_{C W}=11$ and $R e=19,436$ was characterized by a homogenous fibre distribution. The inner bend suspension and the outer bend suspension mean fibre length $l_{1}$ was comparable and the difference $\Delta l_{1, \text { rel }}$ was only $4 \%$ of the feed $l_{l, \text { Feed }}$. Separation 2, $N_{C W}=21$ and $R e=19,206$ was characterized by fibre segregation to the outer bend. The difference in the mean fibre length from the outer bend suspension to the inner bend suspension $\Delta l_{1}$ was $0.335 \mathrm{~mm}$ and $\Delta l_{1, \text { rel }}=22 \%$ of the feed fibre length $l_{1, \text { Feed }}$. $R L A$ (Fig. 4c) was found increased from $h>0.3$. The third case, $N_{C W}=20$ and $R e=25,356$ has no corresponding case in the visual observation study. Higher $R e$ from increased fluid motion can deform or disperse a fibre network. Here I found $\Delta l_{l}=0.262 \mathrm{~mm}$, what is $\Delta l_{1, \text { rel }}=22 \%$ of the feed fibre length $l_{1, \text { Feed }}$. A reduction in the separation quality $\Delta l_{1, \text { rel }}$ compared to case 2 of $20 \%$ for an increase in the treated suspension flow rate of $24 \%$.

Despite the flow splitter design faults, measured differences in the inner bend and outer bend fibrelength distribution (Table 2) agree well with the observation of the fibre aggregation shown in Fig. 3. Further, it is proof, that the observed segregation is preferent for longer fibres and hence full-fills the case of angular length-based fibre segregation as described in the introduction (compare Fig. 1 third row).

\section{Discussion}

The argument of length-based fibre segregation stems from the observed fibre aggregation at the outer bend and a measured increase of $l_{1}$ in the corresponding case. The fibre suspension motion in the cross-section was inaccessible in this study. The nature of the flocculation phenomena will be elucidated based on a comparative literature discussion. Surprisingly, the cross-sectional flow field in coiled pipe flow (Fig. 6: fluid only, corresponding to cases in Redlinger-Pohn et al. (2016a), $R e=3316, \kappa=0.043$ ) and the flow field in a semi-filled horizontal rotating cylinder, i.e. Jacqueline floc generator (Fig. 7, adapted from Soszynski (Soszynski 1987)) show similarities. Both are characterized by a larger mixing vortex which stretches the half-pipe cross-section with respect to the equatorial/horizontal centre plane.

\section{Flow Field in a Coiled Tube Floc-Generator}
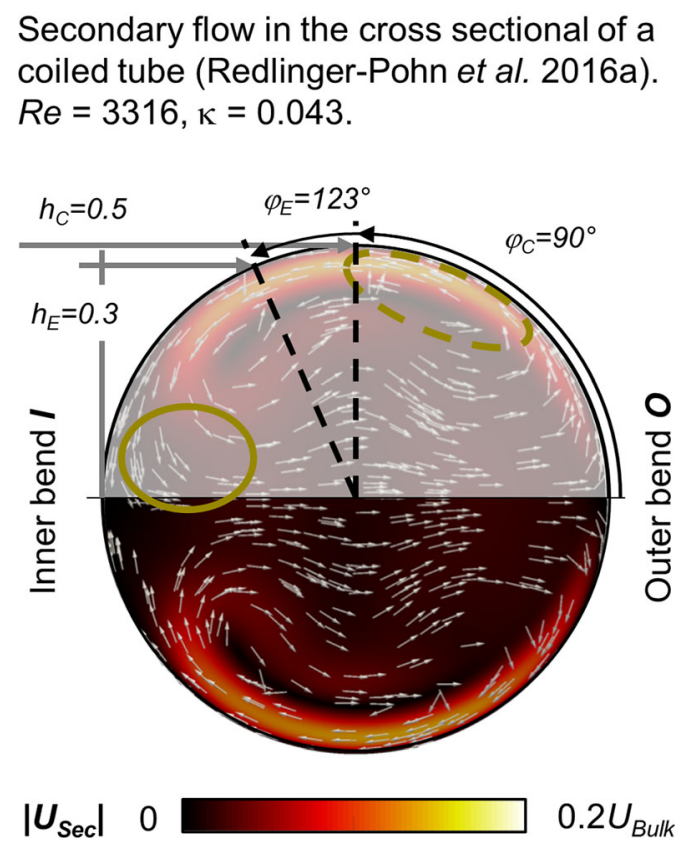

Fig. 6 Secondary motion of coiled pipe flow at $R e=3316$ and $\kappa=0.043$ presented in Redlinger-Pohn et al. (2016a). White arrows present the flow direction and the secondary velocity $U_{S e c}$ magnitude is given by the contour colour. The upper half indicates deacceleration (solid ellipse) and acceleration (dashed ellipse) zones in the cross-section. The upper half is overlaid with the position of fibre segregation $h$ (Fig. 4) and corresponding segment $\varphi$. Indices relate to cases in Fig. 3 


\section{Flow Field in a Horizontal Semi-Filled Cylinder}

Flow field in a horizontal, partially filled rotating cylinder, i.e. Jacqueline floc generator (Soszynski, 1987).

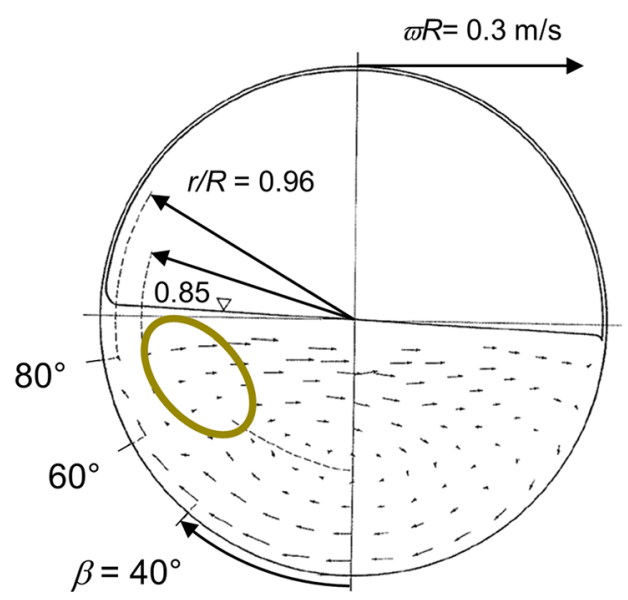

Fig. 7 Secondary motion of a flocculating fibre suspension, in a semi-filled horizontal cylinder, rotating at $\varpi=2 \pi \mathrm{rad} / \mathrm{s}$. The flow field is presented by the arrows. The solid ellipse presents the deacceleration zone with observed flocculation. Figure adapted from Soszynski (Soszynski 1987)

Soszynski (Soszynski 1987) reported for fibre suspension flow in the Jacqueline floc generator:

(i) temporal aggregation of fibres for a concentration below the rigidity threshold $\left(N_{C W}<60\right)$,

(ii) the formation of coherent flocs for higher concentration in the deacceleration zone (marked by a solid ellipse in Fig. 7 at $r /$ $R=0.6$ and angles of $50^{\circ}$ to $90^{\circ}$ ),

(iii) which is characterized by a sharp velocity gradient, i.e. change from upwards-outwards flow to inwards flow.

(iv) The flocs withstood dispersion from hydrodynamic stress when moving through the cylinder cross-section and compacted when re-entering the flocculation zone.

Soszynski (1987) suggested, that a cyclic flow through acceleration and deacceleration zones is required for flocculation. Both zones are present in the secondary motion of the coiled pipe flow (Fig. 6). Focusing on the secondary motion, fibres move through (i) a deacceleration zone at the inner bend centre, and (ii) an acceleration zone at the outer bend wall. Both areas are indicated in Fig. 6, cross-section upper half as full and dashed ellipse, respectively. The position $h$ of changes in RLA (Fig. 4) is drawn on-top of the flow field. $h=0.5$ corresponds to the location of the secondary velocity maximum and $h=0.3$ corresponds to the location of a vortex at the inner bend which directs the suspension towards the tube centre. A clear crowding and floc formation event was not observable from the tube top-view recordings (Fig. 3 and Supplementary Material). But for Case C (Fig. 3c), $N_{C W}=8$, a deformation of flocs at the outer bend and thinning of the fibre flocs at $h \approx 0.5$ was noted. And a high standard deviation in RLA was recorded (Fig. 4b, grey line). The results suggest, that the formed fibre aggregates did not withstand dispersion by the secondary motion fully. Instead, fibres did undergo a dynamic dispersion and aggregation process in the cross-section. For a higher fibre concentration, case $\mathrm{E}$ (Fig. 3e) with a bulk crowding number $N_{C W}$ of 18, no comparable dispersion event was noticed. Additionally, the deformation of the aggregates at the outer bend observed for case $\mathrm{C}$ was not observed for case E.

The mixing flow in a coiled pipe differs from the mixing flow in the Jacqueline floc generator by centrifugal forces acting in direction of the coil radius $R_{\text {Coil }}$ (or $h$ ) and the contribution of an axial (streamwise) flow. Centrifugal forces may lead to sedimentation of the fibres and/or fibre aggregates towards the outer bend in addition to their transportation by the secondary motion. An estimation of scales is provided in the Appendix. The contribution of centrifugal forces increases with the curvature $\kappa$ (which was constant in this study) and the Reynolds number $R e$. For the highest value, i.e. $R e=25,356$, the ratio of the sedimentation velocity from centrifugal forces and the secondary motion at the equatorial centre plane is 0.09 . Hence, centrifugal forces can be neglected and the fibre motion and segregation documented in this study can be attributed to the flow secondary motion. The mixing flow in the coiled pipe is imposed on a stream-wise flow. The maximum velocity of the stream-wise flow is shifted to the outer bend, i.e. $h=0.92$ for the case of $R e=3316$ and $\kappa=0.043$ (Redlinger-Pohn et al. 2016a), and Appendix Fig. 8). Fibre flocs and aggregates moving from the inner bend to the outer bend hence move through an additional acceleration zone into an area of local high shear at the 
outer bend. Fibre aggregates in case $\mathrm{C}$ (shown in Fig. 3c) were accordingly deformed in flow direction. The RLA for case $\mathrm{F}$ is lower than for case $\mathrm{E}$ at the other bend (Fig. 4c) what agrees with a local dispersion of fibres aggregates in the high shear zone at the outer bend.

For straight pipe flow it was shown that for formed fibre networks, the fluid moves at the same speed as the network what results in a plug flow velocity profile (Jäsberg and Kataja 2009; Nikbakht et al. 2014; MacKenzie et al. 2018). Likewise, Soszynski (1987) documented that the flow profile for the case of temporal fibre aggregation and the case of coherent floc formation differed slightly. It is conceivable, that fibre aggregates in the flocculating coiled fibre suspension flow modified the flow field locally. The state of fibre flocculation in straight pipe flow was correlated to the pressure loss curve (see for example (Duffy et al. 1976; Hemström et al. 1976)). Similar measurements can be envisioned to characterize the fibre flocculation in coiled pipe flow and map the flocculation in the $R e-\kappa$ space in future work (see for inspiration the work of Itō (1959) on the friction factor in coiled suspension flow and Canton et al. (2017) for a recent discussion).

The angular segregation was studied below the rigidity threshold $N_{C W}<60$ where non-coherent fibre aggregation is expected. In the regime, the angular segregation benefited from a higher fibre concentration $C$, i.e. increased fibre floc strength and a lower Reynolds number $R e$, i.e. increased dispersion from hydrodynamic stress. That is unique for flocculation from elastic interlocking and different from colloidal flocculation of polymers which also benefits from higher $\operatorname{Re}$ (Carissimi et al. 2018). It raises the question if dispersion, respectively re-suspension, is needed for angular segregation for what it may be limited to the regime of non-coherent flocs, i.e. $N_{C W}<60$. That limit was reported for the treatment of fibre samples injected into coiled pipe flow (Krogerus et al. 2003) and should be addressed for continuous fibre suspension flow in future studies. A second limitation not discussed here is the tube size of $D_{\text {Tube }}<50 \mathrm{~mm}$ (Krogerus et al. 2003). It is conceivable, that the velocity gradient for larger tube diameter as seen by the fibre simply becomes too small to promote flocculation (for example for $l_{1}=1 \mathrm{~mm}$, the ratio $D_{\text {Tube }} / l_{1}=50$ ). Soszynski noted steeper velocity gradients for flocculation in the Jacqueline floc generator as compared to the non-flocculating case (Soszynski 1987). The flow field for flocculation in coiled pipe flow can be addressed in future studies experimentally, for example by magnetic resonance velocimetry (MacKenzie et al. 2018) or numerically as the motion of interacting flexible elongated particles (Tozzi et al. 2005; Schmid et al. 2000; Lindström and Uesaka 2007).

Length-based fibre separation by flow splitting was documented in this study for a fibre suspension propagating 1.75 coil through a tube with $D_{\text {Tube- }}$ $=16 \mathrm{~mm}$ at $\kappa=0.04$ what equals a tube length of $2.2 \mathrm{~m}$. That is by two orders of magnitude shorter than the tube of, for example, 100 m length, used by Silvy and Pascal (1990) and work thereafter. That difference comes from two different attributes of the coiled flow causing angular segregation and axial separation. A scaling analysis is provided in the Appendix. Angular segregation results from mixing in the cross-section and consequent segregation of networked fibres towards the outer bend. The minimum tube length for this study setup was estimated from the Dean vortex turn-over time (i.e. a particle is mixed in the cross-section once) to $1 \mathrm{~m}$. Axial separation results from the stream-wise velocity difference and the fact that fibres aggregated at the outer bend are entrained in the faser flow (see Fig. 9 in the Appendix). The aggregate mean velocity at the outer bend was measured from the recorded images (Fig. 10 in Appendix) to 1.1 times the average velocity. To achieve a good enough separation resolution in the axial direction, i.e. time interval of two consecutive samples of at least $5 \mathrm{~s}$, a $100 \mathrm{~m}$ long tube is needed.

A separation effect in direction of $h$ was recently also documented for rectangular coiled channels. Wang et al. (2020) separated ca. $1 \mu \mathrm{m}$ long $\left(l_{1}\right)$ cellulose-fibrils from smaller fibrils. The longer $1 \mu \mathrm{m}$ fibril was found accumulating at the outer bend of a $45 \mu \mathrm{m}$ high $\left(H_{\text {Channel }}\right)$ and $300 \mu \mathrm{m}$ wide channel. For micro-particles, a separation from the complex interplay of lift-force and drag force from the secondary motion (Ookawara et al. 2006; Di Carlo 2009; Redlinger-Pohn and Radl 2017) is known. Redlinger-Pohn and Radl (Redlinger-Pohn and Radl 2017) have shown that the accumulation at the outer bend appears only for confinements $H_{\text {Channel }} / l_{1}<13$. In Wang et al. (2020) the confinement was $H_{\text {Channel }}$ $l_{1}=45$ and the fibres were highly elongated. As in previous studies on fibre tube flow fractionation, only 
the resulting suspension properties are known but the process itself is a black box. Cross-sectional mixing may also promote aggregation of highly elongated particles in the $\mathrm{nm}$ and $\mu \mathrm{m}$ size range. Certainly, that should be addressed in future studies.

\section{Summary and conclusions}

In this work I documented the fibre flocculation and fibre aggregation in coiled suspension flow at comparable low crowding number, i.e. $N_{C W}=8$ for $R e=$ 9000. The formed aggregates segregated towards the outer bend of the coiled pipe, which presents angular segregation in pipe flow, i.e. fibre accumulation in a circular segment of the tube cross-section. The angular segregation was studied for cases below the rigidity threshold $N_{C W}<60$, hence non-coherent fibre flocs, and a tube diameter of $D_{\text {Tube }}=16 \mathrm{~mm}\left(D_{\text {Tube }} / l_{1 \text {, Fibre- }}\right.$ $=8$ to 10). Literature notes upper limitation for the pipe diameter $\left(D_{\text {Tube }}<50 \mathrm{~mm}\right)$ and fibre concentration. Those should be addressed in future studies. The quality of the angular segregation was probed by flow splitting after 1.75 tube coil (ca. $2 \mathrm{~m}$ tube length). The flow splitter design was based on the observation results. The flow was separated in an inner bend flow and an outer bend flow at $0.3 D_{\text {Tube }}$ in the coil radius $R_{\text {Coil }}$ direction. The spread in mean fibre length $l_{1}$ between outer and inner bend suspension flow was $22 \%$ maximum.

For the studied cases, I found the fibre segregation benefiting from an increased fibre concentration and suffering from increased hydrodynamic stress, i.e. higher flow velocity expressed by a higher Reynolds number. The segregation of fibre aggregates to the outer bend was documented after 1.5 coils, ca. $2 \mathrm{~m}$ but may already be found after 1 coil, i.e. 1 loop, based on an estimated secondary motion turn over time. That raises an interesting question of whether fibre pulp suspensions transported through pipes are mixed or segregated in pipe bents and turns. In this study, I document angular fibre segregation as a promising route for pulp fibre separation by the length. This observation may also explain the documented separation of cellulose nano-fibrils in coiled channel flow (Wang et al. 2020). But some key work for future utilization of coiled pipe flow for the length-based fibre separation remains to be done. The understanding of the fibre flocculation in the Reynolds number $R e$ and curvature $\kappa$ space should be refined. A comparison of the coiled fibre suspension pressure drop to the pressure drop of coiled water flow (Itō 1959) may allow a quantification of the state of flocculation. Phase-contrast magnetic resonance velocimetry (MacKenzie et al. 2018) may allow the direct measurement of the cross-sectional flow profile what allows a detail discussion of the fibre aggregation and dispersion process. The design of the axial flow splitter should be rethought, or replaced by other flowsplitting strategies, for example side-channel splitting as used in hydrodynamic fractionation (RedlingerPohn et al. 2017b).

Acknowledgments Open access funding provided by Royal Institute of Technology. Jakob Redlinger-Pohn thanks the $T^{3} U G$-Teens Treffen Technik internship program at Graz University of Technology for the helping hands of summer intern Veronika Rieger and the financial compensation which sponsored the project. Jakob thanks the Institute of Bioproducts and Paper Technology at Graz University of Technology for the access to their laboratory analytics.

Open Access This article is licensed under a Creative Commons Attribution 4.0 International License, which permits use, sharing, adaptation, distribution and reproduction in any medium or format, as long as you give appropriate credit to the original author(s) and the source, provide a link to the Creative Commons licence, and indicate if changes were made. The images or other third party material in this article are included in the article's Creative Commons licence, unless indicated otherwise in a credit line to the material. If material is not included in the article's Creative Commons licence and your intended use is not permitted by statutory regulation or exceeds the permitted use, you will need to obtain permission directly from the copyright holder. To view a copy of this licence, visit http://creativecommons.org/licenses/by/4.0/.

\section{Appendix}

See Figs. 8, 9, 10.

\section{Notes on the fibre motion}

Table 3 summarizes the fibre motion observation from the video recordings (Supplement Material) for the studied combinations of Reynolds number $R e$ and fibre concentration $C$. The case labeling corresponds to the case labeling in Fig. 3. 

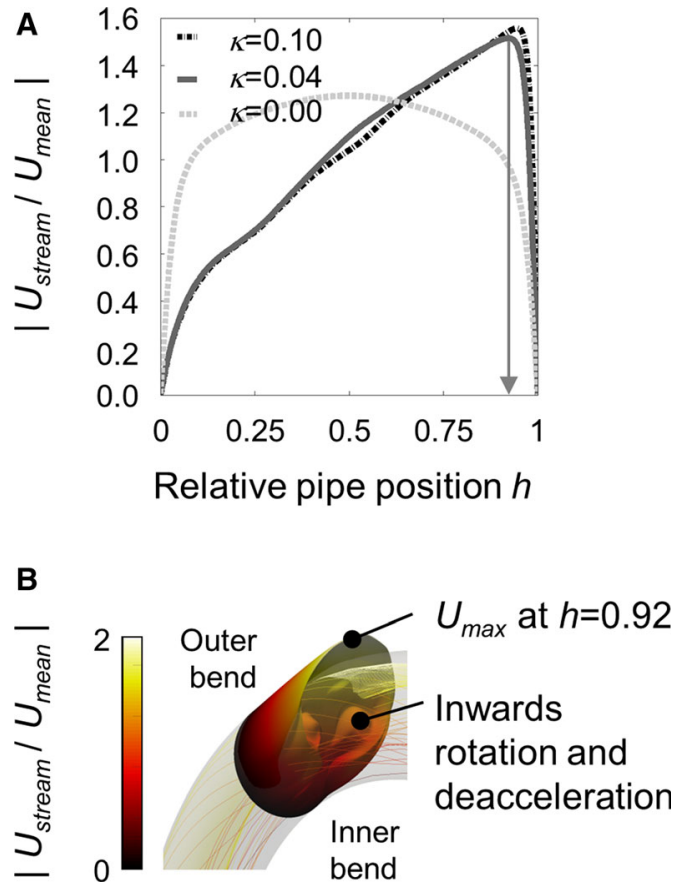

Fig. 8 Coiled pipe velocity profile at $R e=3316$ and increasing curvature $\kappa$. Corresponding to Redlinger-Pohn et al. (2016a). a the stream-wise velocity component $U_{\text {stream }}$ relative to the mean velocity $U_{\text {mean }}$ at the horizontal mid-plane is plotted form the inner bend to the outer bend along $h$. The velocity maximum is deflected to the outer bend for curvature $\kappa=0.04$ and 0.1 and is found at $h=0.92$. b 3D visualization of the coiled pipe flow profile. The stream-wise velocity maximum at the outer bend and mixing vortex at the inner bend is highlighted

\section{Fluid motion in coiled pipe flow}

The curved flow induces centrifugal forces in direction of the coil radius and causes a pressure difference between the inner bend $I$ and the outer bend $O$. The pressure difference gives rise to a secondary motion in a plane normal to the flow direction, i.e. the pipe crosssection. The velocity maximum is shifted from the tube centre toward the outer bend $O$, i.e. $h=0.92$. The change of the streamwise velocity profile along $h$ with the curvature $\kappa$ is presented in Fig. 8a. Figure 8b shows the profile of a coiled pipe flow: secondary motion imposed on the stream-wise flow. Simulation results correspond to cases discussed in RedlingerPohn et al. (2016a): $R e=3316$ and $\kappa=0.00$ (pipe), $\kappa=0.04$ (this work), $\kappa=0.10$.

\section{Top View}

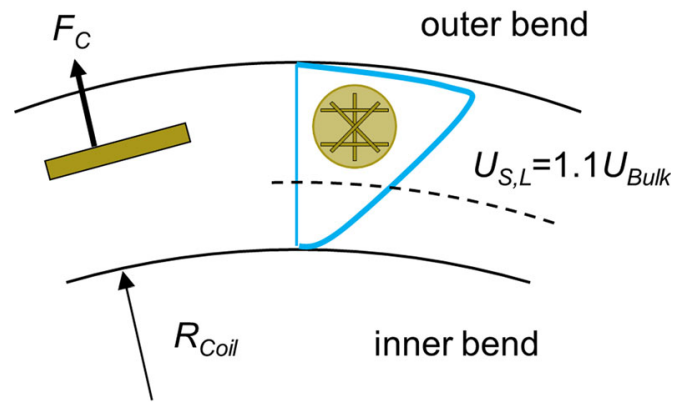

Front View / Cross Section

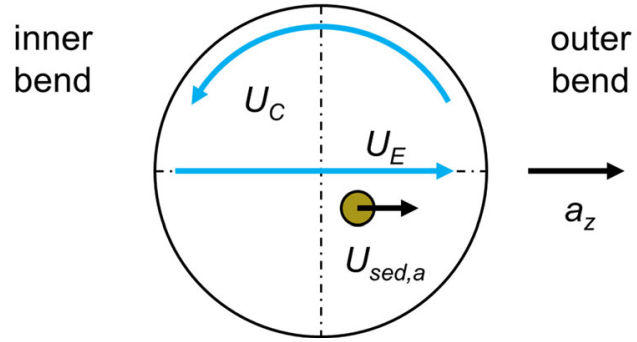

Fig. 9 Relevant velocities and forces on a particle suspended in a coiled flow. $F_{C}$ is the centrifugal force in coil radius direction $R_{\text {Coil }} . U_{S, L}$ is the average velocity at the outer $2 / 3$ rd of the pipe. $U_{E}$ and $U_{C}$ are components of the secondary motion at the equatorial centre plane and along the circumference, respectively. $U_{s e d, a}$ is the sedimentation velocity in the centrifugal acceleration field

\section{Flock Tracking Case C}

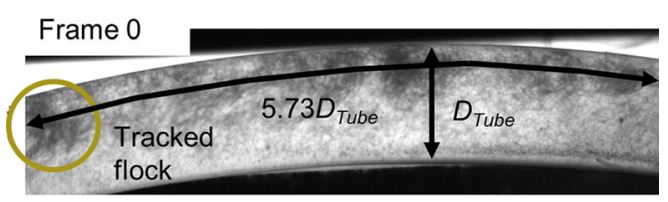

Number of frames. Frame rate $500 \mathrm{~Hz}$.

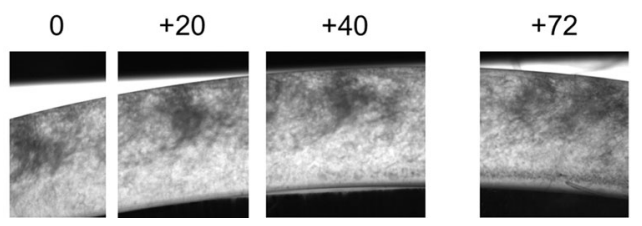

Fig. 10 Propagation speed of a flock, here for case $\mathrm{C}$ ( $R e \approx 9000, \kappa=0.04$, compare to Fig. 3). The tube segment length was measured to $5.73 D_{\text {Tube }}$. The traced fibre flock is pictured after 20, 40, and 72 frames and remained for total 76 frames in the segment 
Table 3 Description of the suspension motion from flow observation. Case labeling corresponds to the labeling in Fig. 3

\begin{tabular}{|c|c|}
\hline Case & $\begin{array}{l}\text { Grainy structures indicating local fibre aggregation. An increase of the grain's numbers was observed towards the outer } \\
\text { bend. } R L A \text { (Fig. } 4 \text { a) was slightly higher at } h=0.8 \text { and smaller for } h<0.5\end{array}$ \\
\hline $\begin{array}{l}\text { Case } \\
\text { B }\end{array}$ & $\begin{array}{l}\text { Grainy structures as observed in case A was not observed in case B. RLA (Fig. 4a) was close to a vertical line what suggests } \\
\text { a homogenous distribution of fibres in the tube }\end{array}$ \\
\hline Case & $\begin{array}{l}\text { Fibres flocs and fibre aggregates were observed at the outer bend. The } R L A \text { was higher for } h>0.7 \text { (Fig. 4b). Fibres are } \\
\text { observed segregated in } h \text { direction and in flow direction: aggregates of fibres at the outer bend were followed by fiber- } \\
\text { depleted suspension. The standard deviation in } R L A \text { is low, despite the identification of crowding fluctuations. A local } \\
\text { fibre crowding was reported Soszynski (1987). Noteworthy is, that fibres aggregated already at a concentration } C \text { that } \\
\text { corresponds to } N_{C W}=8 \text { what is half the gel-point, } N_{C W}=16 \text { (Martinez et al. 2001) }\end{array}$ \\
\hline $\begin{array}{c}\text { Case } \\
\mathrm{D}\end{array}$ & $\begin{array}{l}\text { Fibre flocculation was observed in the tube. The aggregates were not segregated to the outer bend but appeared } \\
\text { in the tube. } R L A \text { (Fig. 4b) shows only little dependence on } h \text {. A slight increase in } R L A \text { is noted at } h=0.6 \text {, } \\
\text { lower for larger and smaller } h\end{array}$ \\
\hline $\begin{array}{c}\text { Case } \\
\text { E }\end{array}$ & $\begin{array}{l}\text { The crowding number } N_{C W}=18 \text { is above the gel-point and the fibre suspension is within the connectivity regime (Celzard } \\
\text { et al. 2009), i.e. the average number of contacts between fibres is larger than } 2 . R L A \text { (Fig. } 4 \mathrm{c} \text { ) was high for } h>0.3 \text {. } \\
\text { Recordings of the inner bend area were over-exposed, and a low fibre concentration at the inner bend might have been } \\
\text { missed. As for case C, segregation in } h \text { and flow direction was observed. The all-over concentration was higher. And from } \\
\text { the video observation no significant motion or deformation of the aggregates within the observation window was noted }\end{array}$ \\
\hline Case F & $\begin{array}{l}\text { As for case } \mathrm{E} \text {, fibres are segregated to the outer bend. The } R L A \text { is comparable to case } \mathrm{E} \text { (Fig. } 4 \mathrm{C} \text { ). Differently, the } \\
\text { segregation in flow direction is less pronounced in case } \mathrm{F} \text { compared to case } \mathrm{E} \text { (Supplement Material). Increased } \\
\text { hydrodynamic stress at higher } R e \text { appears to have a homogenizing effect on the formed fibre flocs }\end{array}$ \\
\hline
\end{tabular}

\section{Centrifugal sedimentation of fibres in pipe flow}

The centrifugal force in $R_{\text {Coil }}$ or $h$ direction differs with the density and leads to sedimentation of heavier particles, for example fibres with a density ratio to water of 1.3 (Redlinger-Pohn et al., 2016a), to the outer bend. The significance of sedimentation in the centrifugal field is estimated from a comparison of the sedimentation velocity in the centrifugal field $U_{\text {sed, } a}$ to the secondary motion at the equatorial centre plane $U_{E}$ (from the inner bend to the outer bend) and along the circumference $U_{C}$ (from the outer bend to the inner bend). The quantities are described in Fig. 9. The magnitude of the secondary motion was estimated from Redlinger-Pohn et al. (2016a) to be $U_{C \text { - }}$ $=0.15 U_{\text {Bulk }}$ and $U_{E}=0.02 U_{\text {Bulk }}$ (see Fig. 6).

The fibre sedimentation velocity can be calculated from the force balance on the fibre,

$0=F_{C}-F_{A}-F_{H}=\left(\rho_{F}-\rho_{W}\right) V_{F} a_{z}-F_{H}$

where $F_{A}$ is the buoyancy force opposite to the centrifugal force $F_{C}$ and $F_{H}$ is the hydrodynamic drag force. $\rho_{F}$ and $\rho_{W}$ are the fibre and water density, respectively. The drag force coefficient $c_{d}$ for a fibre scales with its aspect ratio $A R$ (i.e. the ratio of the fibre length $l$ to the fibre diameter $d$ ) and depends on fibre orientation with respect to the direction of acceleration (see for example (Fan and Ahmadi 1995)). The drag force coefficient for a fibre orientated parallel $\left(c_{d, p}\right)$ and normal $\left(c_{d, n}\right)$ to the direction of acceleration is:

$c_{d, p}=\frac{8\left(A R^{2}-1\right)}{\left[\left(2 A R^{2}-1\right) \ln \left(A R+\left(A R^{2}-1\right)^{0.5}\right) /\left(A R^{2}-1\right)\right]-A R}$

$c_{d, n}=\frac{16\left(A R^{2}-1\right)}{\left[\left(2 A R^{2}-3\right) \ln \left(A R+\left(A R^{2}-1\right)^{0.5}\right) /\left(A R^{2}-1\right)\right]-A R}$

The hydrodynamic drag force is formulated as:

$F_{H}=\eta \pi \frac{l}{2 A R} c_{d} U_{\text {sed }, a}$

$\eta$ is the fluid dynamic viscosity. The orientation of individual fibres in coiled suspension flow was reported by Redlinger-Pohn et al. (2016a) to be in stream-wise direction and hence normal to the direction of sedimentation. Combining Eq. 7 with Eq. 4 and Eq. 6 allows solving for $U_{\text {sed,a }}$. The centrifugal acceleration, $a_{z}=2 U_{\text {Bulk }}^{2} / D_{\text {Coil }}$, is $1.57 \mathrm{~m} / \mathrm{s}^{2}$ and $7.08 \mathrm{~m} / \mathrm{s}^{2}$ for $R e=9000$ and $R e=19,000$, respectively. Estimations (Table 4) are attained for a fibre 
Table 4 Estimation of the fibre sedimentation velocity in the centrifugal field of a coiled suspension flow in comparison to the secondary motion

\begin{tabular}{llllllll}
\hline$R e[-]$ & $U_{\text {Bulk }}[\mathrm{m} / \mathrm{s}]$ & $a_{z}\left[\mathrm{~m} / \mathrm{s}^{2}\right]$ & $U_{C}[\mathrm{~m} / \mathrm{s}]$ & $U_{E}[\mathrm{~m} / \mathrm{s}]$ & $U_{\text {sed,a }}[\mathrm{mm} / \mathrm{s}]$ & $U_{\text {sed, }, a} / U_{C}[\%]$ & $U_{\text {sed, } a} / U_{E}[\%]$ \\
\hline 9000 & 0.56 & 1.57 & 0.084 & 0.011 & 0.353 & 0.42 & 3.15 \\
19,000 & 1.19 & 7.08 & 0.179 & 0.024 & 1.592 & 0.89 & 6.69 \\
25,400 & 1.59 & 12.60 & 0.238 & 0.032 & 2.834 & 1.19 & 8.93 \\
\hline
\end{tabular}

with length $l=5 \mathrm{~mm}$ and an aspect ratio $A R=100$ in water with $\eta=1 \mathrm{mPa}$ s and $\rho_{W}=1000 \mathrm{~kg} / \mathrm{m}^{3}$.

The relative magnitude of sedimentation in the centrifugal field increased with the Reynolds number $R e$ and curvature $\kappa$ as $D_{\text {Coil }}$ decreases. The latter was constant in this study. The impact of sedimentation from centrifugal acceleration at settings in this study is small.

Further, fibres at the studied concentration interact and are not individually suspended. A fibre network or fibre aggregates can be viewed as large porous particles. The aggregate density is close to the density of water. Networks withstanding dispersion can deform (for example consolidation towards the outer bend). The deformation from the centrifugal acceleration needs to be regarded again in relation to the impact of the secondary motion. Fibres locked in the network may have a lower, close to 0 , velocity in $h$ or $R_{\text {Coil }}$ direction. For that, hydrodyamic drag forces from the secondary motion would deform the network. From the estimation in Table 4 can be argued that network deformation form the secondary motion drag force is larger than deformation from the centrifugal force. Hence, fibre motion, aggregation, and dispersion for cases discussed in this paper can be attributed to the secondary motion.

\section{Scaling angular segregation and axial separation in coiled tube flow}

Angular segregation was observed in this study after 1.5 coils what equals a tube length $L_{\text {Tube }}$ of $1.9 \mathrm{~m}$ $\left(L_{\text {Tube }}=1.5 D_{\text {Tube }} \pi \kappa^{-1}\right.$, with $\left.D_{\text {Tube }}=16 \mathrm{~mm}\right)$. That is much shorter than a $100 \mathrm{~m}$ tube used in tube flow fractionation to attain axial separation of a pulp sample (Silvy and Pascal 1990; Laitinen et al. 2011; Jagiello et al. 2016; Redlinger-Pohn et al. 2016a).
Cause for angular segregation is the mixing in the pipe or tube cross-section by the secondary motion. A mixing time $t_{M i x}$ can be estimated for a particle entrained in a Dean vortex, spanning the half crosssection (Dean vortex turn-over time). The particle moves in the cross-section from the outer bend to the inner bend along the tube half-circumference at a speed $U_{C}$ and along the equatorial/horizontal centre plane at a speed of $U_{E}$ (see Fig. 9).

$t_{\text {Mix }}=\frac{D_{\text {Tube }} \pi}{2} \frac{1}{U_{C}}+D_{\text {Tube }} \frac{1}{U_{E}}$

The propagation in stream-wise direction $L_{P}$ can be estimated as the product of the mixing time $t_{M i x}$ and the mean stream-wise velocity $U_{B u l k}$ :

$L_{P}=t_{\text {Mix }} U_{B u l k}=\frac{D_{\text {Tube }} \pi}{2} \frac{U_{\text {Bulk }}}{U_{C}}+D_{\text {Tube }} \frac{U_{\text {Bulk }}}{U_{E}}$

The propagation per tube coil $L_{P, \text { Coil }}$ for one turnover is attained form comparing $L_{P}$ to the coil length, i.e. the circumference of the coil with $D_{\text {Coil }}=k^{-1} D_{\text {Tube }}$.

$L_{P, \text { Coil }}=\frac{L_{P}}{L_{\text {Coil }}}=\frac{L_{P} \kappa}{D_{\text {Tube }} \pi}=\kappa\left(\frac{1}{2} \frac{U_{\text {Bulk }}}{U_{C}}+\frac{1}{\pi} \frac{U_{\text {Bulk }}}{U_{E}}\right)$

With $U_{C}=0.15 U_{B u l k}$ and $U_{E}=0.02 U_{\text {Bulk }}$ propagation length calculates to $L_{P}=1 \mathrm{~m}$ and $L_{P \text {, Coil }}$ of 0.77 (with $D_{\text {Tube }}=16 \mathrm{~mm}$ and $k=0.04$ ). Observations of angular segregation and length-based separation in this paper were attained at approx. twice this value, hence, two theoretical turn-over in the tube cross-section from the secondary mixing motion.

Fibre aggregates segregated to the outer tube bend are entrained in a faster stream-wise flow (see Fig. 8). Hence, the mean stream-wise velocity of longer fibres $U_{S, L}$ is higher than the mean stream-wise velocity of non-aggregated or non-flocculated and in the cross- 
section mixed shorter fibres or fibre-fines $U_{S, F}$. The separation time $t_{T F}$ between fractions scales with the difference in mean stream-wise velocity and the tube length $L_{T F}$ :

$t_{T F}=L_{T F}\left|\frac{1}{U_{S, F}}-\frac{1}{U_{S, L}}\right|$

The fibre aggregates velocity at the outer bend was estimated by tracing fibre flocks in the imaged tube segment (an example is given for case C in Fig. 10. The corresponding video is in the Supplement Material). The tube segment was measured to $5.73 D_{\text {Tube }}$ length and the fibre flock is tracked for 76 frames, taken at a frame rate of $500 \mathrm{~Hz}$.

The propagation velocity calculated to $0.603 \mathrm{~m} / \mathrm{s}$. The bulk velocity at $R e=9000$ is $U_{B u l k}=0.56 \mathrm{~m} / \mathrm{s}$ (Table 4). The relative fibre velocity calculates to $U_{S, L} \approx 1.1 U_{B u l k}$. That agrees with the average streamwise velocity along the equatorial centre line from $h=0.33$ to $h=1$ of ca. $1.13 U_{\text {Bulk }}$ from fluid simulations (Fig. 8). Fibre-fines and small particles can be assumed well mixed in the cross-section and their mean velocity $U_{S, F} \approx 1 U_{B u l k}$. The tube flow fractionation sampling interval $t_{T F}$ in previous work was adjusted to the flow rate being a minimum $5 \mathrm{~s}$ (Jagiello et al. 2016; Redlinger-Pohn et al. 2016a). For a case of $R e=9000$ and $D_{\text {Tube }}=16 \mathrm{~mm}$, the minimum tube length to attain measurable axial separation calculates to $31 \mathrm{~m}$. That is an order of magnitude larger than the propagation length for angular segregation. Further, the axial separation attained by tube flow fractionation is not ideal in long fibres and fibre-fines but in consecutive compartments of decreasing fibre length. Hence, the tube length needs to be appropriate long to achieve attainable axial separation. That may explain the "tube of a few hundred meters" used by Silvy and Pascal (1990) and in succeeding work (Laitinen et al. 2011; Jagiello et al. 2016; Redlinger-Pohn et al. 2016a). Redlinger-Pohn et al. (2016a) reported the relative residence time per fibre fraction $\tau_{L}$ from separation in a $100 \mathrm{~m}$ tube with $D_{\text {Tube }}=16 \mathrm{~mm}$ coiled at $\kappa=0.043$. The Reynolds number was $R e=6926$. The residence time is inversely correlated to the fraction specific mean propagation speed $U_{S, L}$ $\left(U_{B u l k} / U_{S, L}=\tau_{L}\right)$. Table 5 lists $U_{S, L}$ calculated from the fraction residence time in Redlinger-Pohn et al. (2016a) for sulfite pulp, $0.25 \mathrm{wt} \%$ (Redlinger-Pohn et al. 2016a), and $t_{T F}$ as difference from the longest
Table 5 Fibre length fraction, stream-wise propagation velocity per fraction $U_{S \text {, Fraction }}$ and fraction separation time $t_{T F}$. Values from Redlinger-Pohn et al. (2016a)

\begin{tabular}{llll}
\hline$R e[-]$ & $l_{l, \text { Fraction }}[\mathrm{mm}]$ & $U_{S, \text { Fraction }}[\mathrm{m} / \mathrm{s}]$ & $t_{T F}[\mathrm{~s}]$ \\
\hline 6926 & 3.746 & 0.583 & 0 \\
& 1.745 & 0.568 & 4.6 \\
& 0.745 & 0.550 & 10.2 \\
& 0.238 & 0.518 & 21.6 \\
\hline
\end{tabular}

fibre fraction. $t_{T F}$ for fibre-fines after $100 \mathrm{~m}$ separation was measured by them to $21.6 \mathrm{~s}$.

Above, I estimated a tube length of $31 \mathrm{~m}$ for $t_{T F}=5 \mathrm{~s}\left(\right.$ and $\left.U_{S, F}=1.1 U_{B u l k}\right)$. Scaling the separation time $t_{T F}$ to a $100 \mathrm{~m}$ tube and accounting for the different flow rates (scaled by the Reynolds number ratio $9000 / 6926$ as the same viscosity of water and tube diameter $D_{\text {Tube }}$ are used as reference) results to $21.0 \mathrm{~s}$. The axial separation measured from tube flow fractionation (Redlinger-Pohn et al. 2016a) and estimated from angular segregation agree well.

The aggregation of fibres and their angular segregation is quick. The difference in the mean streamwise velocity of the fibre aggregates at the outer bend to the mean bulk velocity is small, and separation in axial direction hence needs residence time within the tube. The tube length for tube flow fractionation, i.e. $100 \mathrm{~m}$, is hence longer than the tube length for flowsplitting after angular segregation, i.e. $1 \mathrm{~m}$. This analysis also suggests that the choice of tube length can tune the separation achieved by tube flow fractionation.

\section{References}

Bossu J, Eckhart R, Czibula C et al (2019) Fine cellulosic materials produced from chemical pulp: the combined effect of morphology and rate of addition on paper properties. Nanomaterials 9:321. https://doi.org/10.3390/ nano9030321

Canton J, Örlü R, Schlatter P (2017) Characterisation of the steady, laminar incompressible flow in toroidal pipes covering the entire curvature range. Int J Heat Fluid Flow 66:95-107. https://doi.org/10.1016/j.ijheatfluidflow. 2017. 05.014

Carissimi E, Sanagiotto DG, Schettini EBC, Rubio J (2018) Revisiting coiled flocculator performance for particle 
aggregation. Water Environ Res 90:322-328. https://doi. org/10.2175/106143017X14902968254935

Celzard A, Fierro V, Kerekes R (2009) Flocculation of cellulose fibres: new comparison of crowding factor with percolation and effective-medium theories. Cellulose 16:983-987. https://doi.org/10.1007/s10570-009-9314-0

Dean WR (1927) XVI. Note on the motion of fluid in a curved pipe. London Edinburgh Dublin Philos Mag J Sci 4:208-223. https://doi.org/10.1080/14786440708564324

Di Carlo D (2009) Inertial microfluidics. Lab Chip 9:3038-3046. https://doi.org/10.1039/b912547g

Duffy GG, Titchener AL, Lee PFW, Moller K (1976) The Mechanisms of Flow of Pulp Suspensions in Pipes. Appita J 29:363-370

Eß1 M (2017) Design of a Distributor for Fibre Suspensions. Graz University of Technology

Fan FG, Ahmadi G (1995) A sublayer model for wall deposition of ellipsoidal particles in turbulent streams. J Aerosol Sci 26:813-840. 8502(95)00021-4

Fischer W, Mayr M, Spirk S et al (2017) Pulp fines-characterization, sheet formation, and comparison to microfibrillated cellulose. Polymers (Basel) 9:366. https://doi.org/ 10.3390/polym 9080366

Hemström G, Moller K, Norman B (1976) Boundary layer studies in pulp suspension flow. TAPPI J 59:115-118

Itō H (1959) Friction Factors for Turbulent Flow in Curved Pipes. J Basic Eng 81:123-132. https://doi.org/10.1115/1. 4008390

Jagiello LA, Redlinger-Pohn JD, Fischer WJ et al (2016) The effect of dean flow in a tube flow fractionation device. Nord Pulp Pap Res J 31:641-647

Jäsberg A, Kataja M (2009) Flow regimes in flows of wood fibre suspensions. In: 14th Pulp Pap. Fundam. Res. Symp. $161-180$

Johansson B, Kubát J (1956) An axial separation effect in flowing pulp suspension. Sven Papp 59:845-846

Jokinen H (2007) Screening and cleaning of pulp — a study to the parameters affecting separation. University of Oulu

Kerekes RJ (2006) Rheology of fibre suspensions in papermaking: An overview of recent research. Nord. Pulp Pap Res J 21:598-612

Kerekes RJ, Schell CJ (1992) Characterization of fibre flocculation regimes by a crowding factor. J Pulp Pap Sci $18: 32-38$

Kerekes RJ, Soszynski RM, Doo T (1985) The flocculation of pulp fibres. In: 8th Fundam. Res. Symp. 265-310

Koutsky JA, Adler RJ (1964) Minimisation of axial dispersion by use of secondary flow in helical tubes. Can J Chem Eng 42:239-246. https://doi.org/10.1002/cjce.5450420602

Krogerus B, Fagerholm K, Löytynoja L (2003) Analytical fractionation of pulps by tube flow. Pap ja Puu 85:209-213

Laitinen O, Kemppainen K, Stoor T, Niinimäki J (2011) Fractionation of pulp and paper particles selectively by size. BioResources 6:672-685

Laitinen O, Löytynoja L, Niinimäki J (2006) Tube flow fractionator-a simple method for laboratory fractionation. Pap ja Puu 88:351-355

Lindström SB, Uesaka T (2007) Simulation of the motion of flexible fibers in viscous fluid flow. Phys Fluids 19:113307. https://doi.org/10.1063/1.2778937
MacKenzie J, Söderberg D, Swerin A, Lundell F (2018) Turbulent stress measurements of fibre suspensions in a straight pipe. Phys Fluids 30:025104. https://doi.org/10. 1063/1.5008395

Martinez DM, Buckley K, Lindström A et al (2001) Characterizing the mobility of papermaking fibres during sedimentation. In: Sci Papermak 12th Fundam Res Symp 16:225-254

Mayr M, Thaller A, Eckhart R, Bauer W (2017) Characterization of fines quality and their independent effect on sheet properties. In: Transactions of the 16Th fundamental research symposium. Oxford, pp 299-322

Meyer H (1964) An analytical treatment of the laminar flow of annulus forming fibrous suspensions in vertical pipes. TAPPI J 47:78-84

Naphon P, Wongwises S (2006) A review of flow and heat transfer characteristics in curved tubes. Renew Sustain Energy Rev 10:463-490. https://doi.org/10.1016/j.rser. 2004.09.014

Niinimäki J, Kumoulainen H, Laitinnen O, Löytynoja L (2007) Method and measuring device for measuring recycled fibre pulp. EP2010902A1.

Nikbakht A, Madani A, Olson JA, Martinez DM (2014) Fibre suspensions in Hagen-Poiseuille flow: transition from laminar plug flow to turbulence. J Nonnewton Fluid Mech 212:28-35. https://doi.org/10.1016/j.jnnfm.2014.08.006

Odabas N, Henniges U, Potthast A, Rosenau T (2016) Cellulosic fines: properties and effects. Prog Mater Sci 83:574-594. https://doi.org/10.1016/j.pmatsci.2016.07.006

Olgard G (1970) Fractionation of fiber suspensions by liquid column flow. TAPPI J 53:1240-1246

Olgard G, Axenfalk SI (1972) Fractionation of pulps with the Johnson Fractionator.pdf. Appita J 26:123-130

Ookawara S, Street D, Ogawa K (2006) Numerical study on development of particle concentration profiles in a curved microchannel. Chem Eng Sci 61:3714-3724. https://doi. org/10.1016/j.ces.2006.01.016

Palazoglu TK, Sandeep KP (2004) Effect of tube curvature ratio on the residence time distribution of multiple particles in helical tubes. LWT Food Sci Technol 37:387-393. https:// doi.org/10.1016/S0023-6438(03)00013-6

Redlinger-Pohn JD, Radl S (2017) Size-based particle separation in coiled channel flow of non-circular cross-section. In: Proceedings of the 27th European Symposium on Computer Aided Process Engineering - ESCAPE 27.

Redlinger-Pohn JD, Jagiello LA, Bauer W, Radl S (2016a) Mechanistic understanding of size-based fiber separation in coiled tubes. Int J Multiph Flow. https://doi.org/10.1016/j. ijmultiphaseflow.2016.04.008

Redlinger-Pohn JD, König LM, Kloss C, et al (2016b) Modeling of non-spherical, elongated particles for industrial suspension flow simulation. In: ECCOMAS Congress 2016proceedings of the 7 th european congress on computational methods in applied sciences and engineering

Redlinger-Pohn JD, Bauer W, Radl S (2017a) Fractionation of fibre pulp in a hydrodynamic fractionation device: influence of reynolds number and accept flow rate. In: Trans 16Th Fundam Res Symp Held Oxford Sept 2017 209-228

Redlinger-Pohn JD, König J, Radl S (2017) Length-selective separation of cellulose fibres by hydrodynamic 
fractionation. Chem Eng Res Des. https://doi.org/10.1016/ j.cherd.2017.08.001

Schmid CF, Switzer LH, Klingenberg DJ (2000) Simulations of fiber flocculation: Effects of fiber properties and interfiber friction. J Rheol (N Y N Y) 44:781-809. https://doi.org/10. $1122 / 1.551116$

Schmid T, Redlinger-Pohn JD, Radl S (2019) Length-based hydrodynamic fractionation of highly networked fibers in a mini-channel. Nord Pulp Pap Res J 34:182-199. https:// doi.org/10.1515/npprj-2018-0086

Silvy J, Pascal M (1990) Device for determining the characteristics of particles in suspension in a liquid. US005087823A

Soszynski R (1987) The formation and properties of coherent flocs in fibre suspensions. University of British Columbia

Soszynski RM, Kerekes RJ (1988a) Elastic interlocking of nylon fibers suspended in liquid. Part 1. Nature of cohesion among fibers. Nord Pulp Pap Res J 3:172-179. https://doi. org/10.3183/npprj-1988-03-04-p172-179

Soszynski RM, Kerekes RJ (1988b) Elastic interlocking of nylon fibers suspended in liquid. Part 2. Process of interlocking. Nord Pulp Pap Res J 3:180-184. https://doi.org/ 10.3183/npprj-1988-03-04-p180-184
Steenberg B, Wahren D (1960) Concentration gradients in boundary layers of streaming fibre suspensions. Sven Papp 63:347-355

Talimi V, Muzychka YS, Kocabiyik S (2012) A review on numerical studies of slug flow hydrodynamics and heat transfer in microtubes and microchannels. Int J Multiph Flow 39:88-104. https://doi.org/10.1016/j. ijmultiphaseflow.2011.10.005

Tozzi EJ, Klingenberg DJ, Scott CT, Miettinen P (2005) Simulations of flexible fiber suspensions. In: Proceedings of the 2005 TAPPI Practical Papermaking Conference : 2005 May 22-26, Milwaukee, WI

Vashisth S, Kumar V, Nigam KDP (2008) A review on the potential applications of curved geometries in process industry. Ind Eng Chem Res 47:3291-3337. https://doi.org/ 10.1021/ie701760h

Wang X, Li R, Zeng J et al (2020) Efficient fractionation of cellulose nanofibers using spiral microchannel. Cellulose. https://doi.org/10.1007/s10570-020-03072-2

Publisher's Note Springer Nature remains neutral with regard to jurisdictional claims in published maps and institutional affiliations. 